\title{
Effects of Viscous Dissipation on MHD Natural Convection Flow along a Vertical Wavy Surface with Heat Generation
}

\author{
K. H. Kabir ${ }^{1}$, M. A. Alim² ${ }^{2}$ L. S. Andallah ${ }^{3}$ \\ ${ }^{1}$ Department of Mathematics, Mohammadpur Kendriya University College, Dhaka, Bangladesh \\ ${ }^{2}$ Department of Mathematics, Bangladesh University of Engineering and Technology, Dhaka, Bangladesh \\ ${ }^{3}$ Department of Mathematics, Jahangirnagar University, Savar, Bangladesh \\ Email:kfzkabir@gmail.com,a0alim@gmail.com, andallahls@gmail.com
}

Received February 16, 2013; revised March 20, 2013; accepted April 17, 2013

Copyright (C) 2013 K. H. Kabir et al. This is an open access article distributed under the Creative Commons Attribution License, which permits unrestricted use, distribution, and reproduction in any medium, provided the original work is properly cited.

\begin{abstract}
The effect of external magnetic field and internal heat generation or absorption on a steady two-dimensional natural convection flow of viscous incompressible fluid along a uniformly heated vertical wavy surface has been investigated. The governing boundary layer equations are first transformed into a non-dimensional form using suitable set of dimensionless variables. The transformed boundary layer equations are solved numerically using the implicit finite difference method, known as Keller-box scheme. Numerical results for velocity, temperature, skin friction, the rate of heat transfer are obtained for different values of the selected parameters, such as viscous dissipation parameter $(V d)$, heat generation parameter $(Q)$, magnetic parameter $(M)$ and presented graphically and discussed. Streamlines and isotherms are presented for selected values of heat generation parameter and explained.
\end{abstract}

Keywords: Natural Convection; Wavy Surface; Magnetohydrodynamics; Viscous Dissipation; Prandtl Number; Heat Generation

\section{Introduction}

The natural convection boundary layer flow about a heated vertical wavy surface has received a great deal of attention due to its relation to practical applications of complex geometries. There is also a model problem for the investigation of heat transfer from roughened surfaces in order to understand heat transfer enhancement. The natural convection along a vertical wavy surface was first studied by Yao [1] and used an extended Prantdl's transposition theorem and a finite-difference scheme. He proposed a simple transformation to study the natural convection heat transfer from isothermal vertical wavy surfaces, such as sinusoidal surface. Moulic and Yao [2] also investigated mixed convection heat transfer along a vertical wavy surface. Alam et al. [3] have also studied the problem of free convection from a wavy vertical surface in presence of a transverse magnetic field. The combined effects of thermal and mass diffusion on the natural convection flow of a viscous incompressible fluid along a vertical wavy surface have been investigated by Hossain and Rees [4]. Wang and Chen [5] investigated tran- sient force and free convection along a vertical wavy surface in micropolar fluid. Hossain et al. [6] have studied the problem of natural convection of fluid with temperature dependent viscosity along a heated vertical wavy surface. Natural and mixed convection heat and mass transfer along a vertical wavy surface have been investigated by Jang [7,8]. Recently, Molla et al. [9] have studied natural convection flow along a vertical wavy surface with uniform surface temperature in presence of heat generation/absorption. Tashtoush and Al-Odat [10] investigated magnetic field effect on heat and fluid flow over a wavy surface with a variable heat flux. Hossain [11] investigated the natural convection flow past a permeable wedge for the fluid having temperature dependent viscosity and thermal conductivity. Very recently, Parveen and Alim [12] investigated Joule heating effect on Magnetohydrodynamic natural convection flow along a vertical wavy surface with viscosity dependent on temperature. The present study is to incorporate the effects of the viscous dissipation on MHD natural convection flow along a uniformly heated vertical wavy surface with heat generation. 
Numerical results have been obtained in terms of local skin friction coefficient and the rate of heat transfer in terms of local Nusselt number, and the velocities as well as the temperature profiles for a selection of relevant physical parameters are shown graphically.

\section{Formulation of the Problem}

Steady two dimensional laminar free convection boundary layer flow of a viscous incompressible and electrically conducting fluid along a vertical wavy surface in presence of uniform transverse magnetic field is considered. It is assumed that the wavy surface is electrically insulated and is maintained at a uniform temperature $T_{w}$. The fluid is stationary above the wavy plate and is kept at a temperature $T_{\infty}$. The surface temperature $T_{w}$ is greater than the ambient temperature $T_{\infty}$ that is, $T_{w}>T_{\infty}$. The flow configuration of the wavy surface and the two-dimensional cartesian coordinate system are shown in Figure 1.

The boundary layer analysis outlined below allows $\bar{\sigma}(X)$ being arbitrary, but our detailed numerical work assumed that the surface exhibits sinusoidal deformations. The wavy surface may be defined by

$$
Y_{w}=\bar{\sigma}(X)=\alpha \sin \left(\frac{n \pi X}{L}\right)
$$

where $\alpha$ is the amplitude and $L$ is the wave length associated with the wavy surface.

The governing equations of such flow of magnetic field in presence of heat generation/absorption with viscosity variation along a vertical wavy surface under the usual Boussinesq approximations can be written in a dimensional form as:

Continuity Equation

$$
\frac{\partial U}{\partial X}+\frac{\partial V}{\partial Y}=0
$$

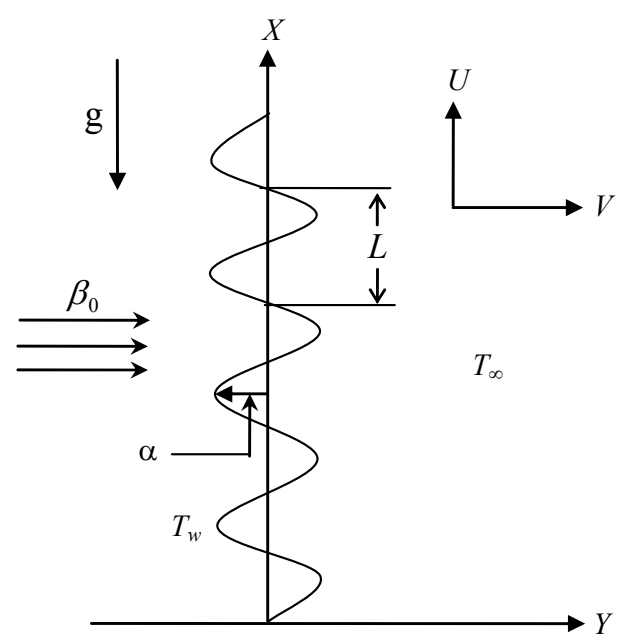

Figure 1. Physical model and coordinate system.

$$
\begin{aligned}
U \frac{\partial U}{\partial X}+V \frac{\partial U}{\partial Y}= & -\frac{1}{\rho} \frac{\partial P}{\partial X}+\frac{1}{\rho} \nabla \cdot(\mu \nabla U) \\
& +g \beta\left(T-T_{\infty}\right)-\frac{\sigma_{0} \beta_{0}^{2}}{\rho} U \\
U \frac{\partial V}{\partial X}+V \frac{\partial V}{\partial Y}= & -\frac{1}{\rho} \frac{\partial P}{\partial Y}+\frac{1}{\rho} \nabla \cdot(\mu \nabla V) \\
U \frac{\partial T}{\partial X}+V \frac{\partial T}{\partial Y}= & \frac{k}{\rho C_{P}} \nabla^{2} T+\frac{v}{\rho C_{P}}\left(\frac{\partial U}{\partial Y}\right)^{2} \\
& +\frac{Q_{o}}{\rho C_{p}}\left(T-T_{\infty}\right)
\end{aligned}
$$

where $(X, Y)$ are the dimensional coordinates along and normal to the tangent of the surface and $(U, V)$ are the velocity components parallel to $(X, Y), g$ is the acceleration due to earth gravity, $P$ is the dimensional pressure of the fluid, $T$ is the temperature of the fluid in the boundary layer, $C_{P}$ is the specific heat at constant pressure, $\mu$ is the dynamic viscosity of the fluid in the boundary layer region depending on the fluid temperature, $\rho$ is the density, $v$ is the kinematic viscosity, where $v=\mu / \rho, k$ is the thermal conductivity of the fluid, $\beta$ is the volumetric coefficient of thermal expansion, $\beta_{0}$ is the strength of magnetic field, $\sigma_{0}$ is the electrical conductivity of the fluid and $\nabla^{2}$ is the Laplacian operator, where $\nabla^{2}=\frac{\partial^{2}}{\partial x^{2}}+\frac{\partial^{2}}{\partial y^{2}}$.

The boundary conditions for the present problem are

$$
\begin{aligned}
& U=0, V=0, T=T_{w} \text { at } Y=Y_{w}=\bar{\sigma}(X) \\
& U=0, T=T_{\infty}, P=p_{\infty} \text { as } Y \rightarrow \infty
\end{aligned}
$$

Using Prandtl's transposition theorem to transform the irregular wavy surface into a flat surface as extended by Yao [1] and boundary layer approximation, the following dimensionless variables are introduced for non-dimensionalizing the governing equations

$$
\begin{aligned}
& x=\frac{X}{L}, y=\frac{Y-\bar{\sigma}}{L} G r^{\frac{1}{4}}, p=\frac{L^{2}}{\rho v^{2}} G r^{-1} P, \\
& u=\frac{\rho L}{\mu_{\infty}} G r^{-1 / 2} U, v=\frac{\rho L}{\mu_{\infty}} G r^{-1 / 4}\left(V-\sigma_{x} U\right), \\
& \theta=\frac{T-T_{\infty}}{T_{w}-T_{\infty}}, \sigma_{x}=\frac{\mathrm{d} \bar{\sigma}}{\mathrm{d} X}=\frac{\mathrm{d} \sigma}{\mathrm{d} x}, \\
& G r=\frac{g \beta\left(T_{w}-T_{\infty}\right)}{v^{2}} L^{3}
\end{aligned}
$$

where $\theta$ is the dimensionless temperature function and $(u, v)$ are the dimensionless velocity components parallel to $(x, y)$ and $G r$ is the Grashof number. Now introducing the dimensionless dependent and independent variables into Equations (2)-(5), and the following di- 
mensionless form of the governing equations is obtained after ignoring terms of smaller orders of magnitude in the Grashof number $G r$.

$$
\begin{aligned}
& \frac{\partial u}{\partial x}+\frac{\partial v}{\partial y}=0 \\
& u \frac{\partial u}{\partial x}+v \frac{\partial u}{\partial y}=-\frac{\partial p}{\partial x}+G r^{1 / 4} \sigma_{x} \frac{\partial p}{\partial y} \\
& +\left(1+\sigma_{x}^{2}\right) \frac{\partial^{2} u}{\partial y^{2}}-M u+\theta \\
& \sigma_{x}\left(u \frac{\partial u}{\partial x}+v \frac{\partial u}{\partial y}\right) \\
& =-G r^{1 / 4} \frac{\partial p}{\partial y}+\sigma_{x}\left(1+\sigma_{x}^{2}\right) \frac{\partial^{2} u}{\partial y^{2}}-\sigma_{x x} u^{2} \\
& u \frac{\partial \theta}{\partial x}+v \frac{\partial \theta}{\partial y}=\frac{1}{\operatorname{Pr}}\left(1+\sigma_{x}^{2}\right) \frac{\partial^{2} \theta}{\partial y^{2}}+v d\left(\frac{\partial u}{\partial y}\right)^{2}+Q \theta
\end{aligned}
$$

It is worth noting that the $\sigma_{x}$ and $\sigma_{x x}$ indicate the first and second derivatives of $\sigma$ with respect to $x$, therefore,

$$
\sigma_{x}=\mathrm{d} \bar{\sigma} / \mathrm{d} X=\mathrm{d} \sigma / \mathrm{d} x \text { and } \sigma_{x x}=\mathrm{d} \sigma_{x} / \mathrm{d} x .
$$

In the above equations $\operatorname{Pr}, V d, M$ and $Q$ are respectively known as the Prandtl number, viscous dissipation parameter, magnetic parameter and heat generation parameter which are defined as:

$$
\begin{aligned}
& \operatorname{Pr}=\frac{C_{p} \mu_{\infty}}{k}, V d=\frac{G r}{L^{2} C_{p}\left(T_{w}-T_{\infty}\right)}, \\
& M=\frac{\sigma_{0} \beta_{0}^{2} L^{2}}{\mu G r^{1 / 2}}, Q=\frac{Q_{o} L^{2}}{\mu C_{p} G r^{\frac{1}{2}}}
\end{aligned}
$$

For the present problem the pressure gradient $(\partial p / \partial x=0)$ is zero. Thus, the elimination of $\partial p / \partial y$ from Equations (9) and (10) leads to

$$
\begin{aligned}
u \frac{\partial u}{\partial x}+v \frac{\partial u}{\partial y}= & \left(1+\sigma_{x}^{2}\right) \frac{\partial^{2} u}{\partial y^{2}}-\frac{\sigma_{x} \sigma_{x x}}{1+\sigma_{x}^{2}} u^{2} \\
& -\frac{M}{1+\sigma_{x}^{2}} u+\frac{1}{1+\sigma_{x}^{2}} \theta
\end{aligned}
$$

The corresponding boundary conditions for the present problem then turn into

$$
\left.\begin{array}{ll}
u=v=0, \theta=1, & \text { at } y=0 \\
u=\theta=0, & \text { as } y \rightarrow \infty
\end{array}\right\}
$$

Now we introduce the following transformations to reduce the governing equations to a convenient form:

$$
\psi=x^{3 / 4} f(x, \eta), \eta=y x^{-1 / 4}, \theta=\theta(x, \eta)
$$

where $f(x, \eta)$ is the dimensionless stream function, $\eta$ is the dimensionless similarity variable and $\psi$ is the stream function that satisfies the continuity Equation (8) and is related to the velocity components in the usual way as

$$
u=\frac{\partial \psi}{\partial y}, v=-\frac{\partial \psi}{\partial x}
$$

Introducing the transformations given in Equation (15) and using (16) into Equations (13) and (11) are transformed into the new co-ordinate system. Thus the resulting equations are

$$
\begin{aligned}
& \left(1+\sigma_{x}^{2}\right) f^{\prime \prime \prime}+\frac{3}{4} f f^{\prime \prime}-\left(\frac{1}{2}+\frac{x \sigma_{x} \sigma_{x x}}{1+\sigma_{x}^{2}}\right) f^{\prime 2} \\
& +\frac{1}{1+\sigma_{x}^{2}} \theta-\frac{M x^{1 / 2}}{1+\sigma_{x}^{2}} f^{\prime}=x\left(f^{\prime} \frac{\partial f^{\prime}}{\partial x}-f^{\prime \prime} \frac{\partial f}{\partial x}\right) \\
& \frac{1}{\operatorname{Pr}}\left(1+\sigma_{x}^{2}\right) \theta^{\prime \prime}+\frac{3}{4} f \theta^{\prime}+V d x\left(f^{\prime \prime}\right)^{2}+x^{1 / 2} Q \theta \\
& =x\left(f^{\prime} \frac{\partial \theta}{\partial x}-\theta^{\prime} \frac{\partial f}{\partial x}\right)
\end{aligned}
$$

The boundary conditions (14) now take the following form:

$$
\left.\begin{array}{l}
f(x, 0)=f^{\prime}(x, 0)=0, \theta(x, 0)=1 \\
f^{\prime}(x, \infty)=0, \theta(x, \infty)=0
\end{array}\right\}
$$

Here prime denote the differentiation with respect to $\eta$.

However, once we know the values of the functions $f$ and $\theta$ and their derivatives, it is important to calculate the values of the rate of heat transfer in terms of local Nusselt number $N u_{x}$ and the shearing stress $\tau_{w}$ in terms of the local skin friction coefficient $C_{f x}$ from the following relations:

$$
N u_{x}=\frac{q_{w} X}{k\left(T_{w}-T_{\infty}\right)}, C_{f x}=\frac{2 \tau_{w}}{\rho U^{2}}
$$

where

$$
\begin{aligned}
& q_{w}=-k(\bar{n} \cdot \nabla T)_{y=0}, \\
& \tau_{w}=(\mu \bar{n} \cdot \nabla \bar{u})_{y=0}, \\
& U=\mu_{\infty} G r^{1 / 2} / \rho L .
\end{aligned}
$$

Here $\bar{n}=\frac{\bar{i} f_{x}+\bar{j} f_{y}}{\sqrt{f_{x}^{2}+f_{y}^{2}}}$ is the unit normal to the surface.

Using the transformation (15) and (21) into Equation (20) the local skin friction coefficient $C_{f x}$ and the rate of heat transfer in terms of the local Nusselt number $N u_{x}$ take the following forms:

$$
\begin{aligned}
& \frac{1}{2}(G r / x)^{1 / 4} C_{f x}=\sqrt{1+\sigma_{x}^{2}} f^{\prime \prime}(x, 0) \\
& G r^{-1 / 4} x^{-3 / 4} N u_{x}=-\sqrt{1+\sigma_{x}^{2}} \theta^{\prime}(x, 0)
\end{aligned}
$$


For the computational purpose the period of oscillations in the waviness of this surface has been considered to be $\pi$.

\section{Results and Discussion}

We have investigated the effects of viscous dissipation on natural convection flow of viscous incompressible fluid along a uniformly heated vertical wavy surface. Although there are five parameters of interest in the present problem, the effects of Prandtl number Pr, viscous dissipation $V d$, magnetic parameter $M$, the heat generation parameter $Q$ and the amplitude of the wavy surface $\alpha$ on the surface shear stress in terms of local skin friction coefficient, the rate of heat transfer in terms of the local Nusselt number, the velocity and temperature profiles, the streamlines and the isotherms. Numerical values of local shearing stress and the rate of heat transfer are calculated from Equations (22) and (23) in terms of the skin-friction coefficients $C_{f x}$ and Nusselt number $N u_{x}$ respectively for a wide range of the axial distance variable $x$ starting from the leading edge for different values of the parameters $\operatorname{Pr}, V d, M, Q$ and $\alpha$. Solutions are obtained in terms of velocity profiles, temperature profiles against $\eta$ and the skin friction coefficients $C_{f x}$, the rate of heat transfer in terms of the Nusselt number $N u_{x}$ at any position of $x$ presented graphically for selected values of magnetic parameter $M=0.0,0.5,1.5,2.5,5.0$, viscous dissipation parameter $V d=0.0,5.0,10.0,20.0,30.0$ and heat generation parameter $Q=0.0,0.5,1.0,1.5,2.0$.

The effects for different values of magnetic parameter $M$ on the velocity and temperature profiles with $\alpha=0.2$, $\operatorname{Pr}=0.72, V d=10.0$ and $Q=0.4$ have been presented graphically in Figures 2(a) and (b). It is seen from the Figures 2(a) that for the values of magnetic parameter $M$ $=0.0,0.5,1.5,2.5,5.0$ the velocity decreasing upto the position of $\eta=5.5$ from the wall. At the position of

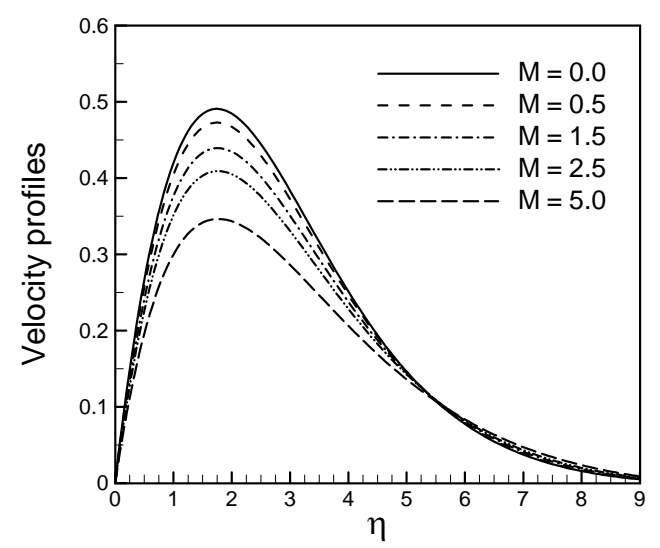

(a) $\eta=5.5$ velocity becomes constant that is velocity profiles meet at a point and then cross the side and increasing with magnetic parameter $M$. This is because of the velocity profiles having lower peak values for higher values of magnetic parameter $M$ tend to decreases comparatively slower along $\eta$-direction than velocity profiles with higher peak values for lower values of magnetic parameter $M$. The maximum values of velocities are recorded as $0.49091,0.47285,0.43933,0.40912$ and 0.34643 for magnetic parameter $M=0.00,0.50,1.50$, $2.50,5.00$ respectively which occur at the same position $\eta=1.73814$. Here, it is observed that at $\eta=1.73814$, the maximum velocity decreases by $29.43 \%$ as the magnetic parameter $M$ change from 0.0 to 5.0. The values of temperature are recorded as $0.70911,0.71666,0.73133$, 0.74536 and 0.77742 for magnetic parameter $M=0.00$, $0.50,1.50,2.50,5.00$ at the same position of $\eta=$ 1.23788 and the temperature increases by $9.63 \%$. In Figures 3(a) and (b) the effects for different values of the viscous dissipation parameter $V d$ on the velocity and temperature profiles with $\alpha=0.2, \operatorname{Pr}=0.72, M=0.1$ and $Q=0.4$ have been shown graphically. It has been seen from Figure 3(a) that as the viscous dissipation parameter $V d$ increases, the velocities rising up to the position of $\eta=1.73814$ for viscous dissipation parameter $V d=0.0,5.0,10.0,20.0,30.0$ and from that position of $\eta$ velocities fall down slowly and finally approaches to zero. It is also observed from Figure 3(b) that as the viscous dissipation parameter $V d$ increases, the temperature profiles increases. The maximum values of velocities are recorded as $0.48686,0.48888,0.49091,0.49499$ and 0.49909 for viscous dissipation parameter $V d=0.0$, $5.0,10.0,20.0,30.0$ respectively which occur at the same position $\eta=1.73814$ and the maximum velocity increases by $2.51 \%$. Temperatures are recorded as 0.69855 , $0.70380,0.70911,0.71991$ and 0.73095 for viscous dissipation parameter $V d=0.0,5.0,10.0,20.0,30.0$ respec-

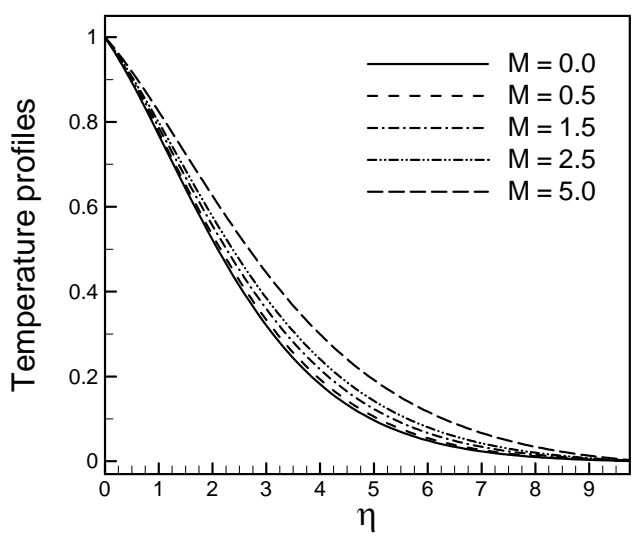

(b)

Figure 2. (a) Velocity and (b) Temperature profiles against $\eta$ for different values of $M$ with $\operatorname{Pr}=0.72, \alpha=0.2, V d=10.0$ and $Q=0.4$. 


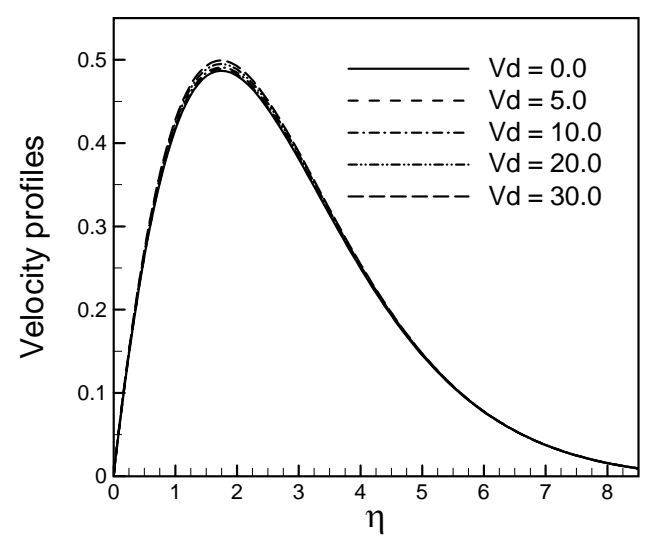

(a)

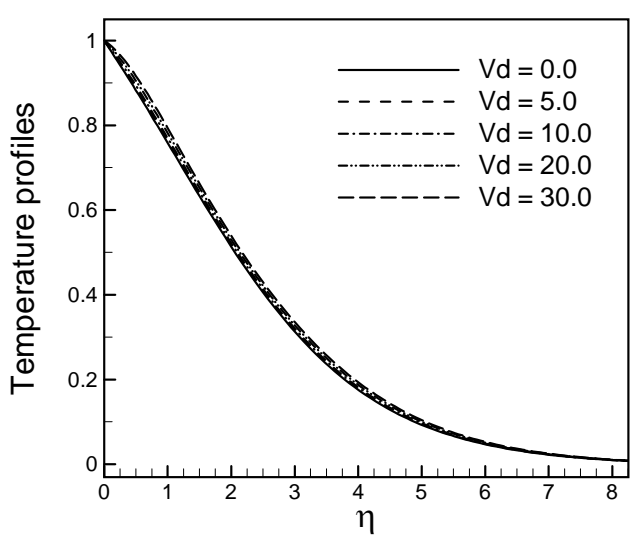

(b)

Figure 3. (a) Velocity and (b) Temperature profiles against $\eta$ for different values of $V d$ with $\operatorname{Pr}=0.72, \alpha=0.2, M=0.1$ and $Q$ $=0.4$.

tively at the same position of $\eta=1.23788$ and the temperature profiles increases by $4.64 \%$. Both the velocity and temperature profiles accumulate nearly in the following points where $\eta=7.55722$ and $\eta=7.86828$ respectively for viscous dissipation parameter $V d=0.0$, $5.0,10.0,20.0,30.0$. That is, velocity boundary layer thickness and thermal boundary layer thickness are unchanged. The effects for different values of the heat generation parameter $Q=0.0,0.5,1.0,1.5,2.0$ on the velocity and temperature profiles with $\alpha=0.2, \operatorname{Pr}=0.72$, $M=0.1$ and $V d=10.0$ have been presented graphically in Figures 4(a) and (b) respectively. For the higher values of the heat generation parameter $Q$ both the velocity and the temperature increases.

In Figures 5(a) and (b) effects of magnetic parameter $M=0.0,0.5,1.5,2.5,5.0$ on skin friction and the rate of heat transfer with $\alpha=0.2, \operatorname{Pr}=0.72, V d=10.0$ and $v$ have been presented. From Figure 5(a) it is found that skin friction decreases significantly for greater magnetic field strength. This is physically realizable as the magnetic field retards the velocity field and consequently reduces the frictional force at the wall. However rate of heat transfer opposite pattern due to the higher values of magnetic parameter $M$ which are presented in Figure 5(b). The different values of viscous dissipation parameter $V d$ with $\alpha=0.2, \operatorname{Pr}=0.72, M=0.1$ and $Q=0.4$. of the skin friction coefficients and the rate of heat transfer are shown graphically in Figures 6(a) and (b) respectively. In this case the values of local skin friction coefficient $C_{f x}$ are recorded to be $1.12663,1.48796,2.03818$, 4.01586 and 7.94542 for $V d=0.0,5.0,10.0,20.0,30.0$ which occur at same point $x=1.51$. From the Figure 6(a), it is observed that at $x=1.51$, the skin friction coefficient increases by $605.23 \%$ due to the higher value of viscous dissipation parameter $V d$. However, the values of rate of heat transfer are found to be $-0.07240,-3.63124$, $-11.82060,-66.89554$, and -284.88541 for $V d=0.0,5.0$,
10.0, 20.0, 30.0 which occur at same point $x=1.51$. The rate of heat transfer coefficient decreases by $393.39 \%$ due to the increased value of viscous dissipation parameter $V d=0.0,5.0,10.0,20.0,30.0$. It is seen from the Figure 6(b) that for higher values of viscous dissipation parameter the rate of heat transfer decreases that is heat transfer slows down for higher viscous dissipation parameter $V d$. In Figures 7(a) and (b) the skin friction coefficient $C_{f x}$ and local rate of heat transfer $N u_{x}$ for different values of heat generation parameter $Q=0.0,0.5$, $1.0,1.5,2.0$ with $\alpha=0.2, \operatorname{Pr}=0.72, M=0.1$ and $V d=$ 10.0 have been displayed. It is observed from the Figure 7(a) that for higher values of heat generation parameter $Q$, skin friction increasing up to the axial position of $x=$ 0.6 and then skin friction becomes constant for all values of $Q$ that is, skin friction coefficient meet together at the position of $x=0.6$ and cross the sides that means after the axial position of $x=0.6$ skin friction is decreasing for higher values of heat generation parameter but frictional force at the wall always rising towards downstream. It is seen from the Figure 7(b) that for higher values of heat generation parameter the rate of heat transfer decreases down to the axial position of $x=1.3$ and then rate of heat transfer becomes constant for all values of $Q$ that is, rate of heat transfer meet together at the position of $x=1.3$ and cross the sides that means after the axial position of $x$ $=1.3$ rate of heat transfer is increasing up for higher values of heat generation.

In Figure 8(a) and (b) are shown that the effects of streamlines and isotherms for different values of heat generation parameter $Q=0.0$ and 2.0 with amplitude of waviness of the surface $\alpha=0.2$, Prandtl number $\operatorname{Pr}=$ 0.72 , magnetic parameter $M=0.1$ and viscous dissipation parameter $V d=10.0$ respectively. The value of stream function $\psi$ is 0.00 near the wall and then $\psi$ increases gradually within the boundary layer and away from the wall. On the other hand the isolines of temperature (iso- 


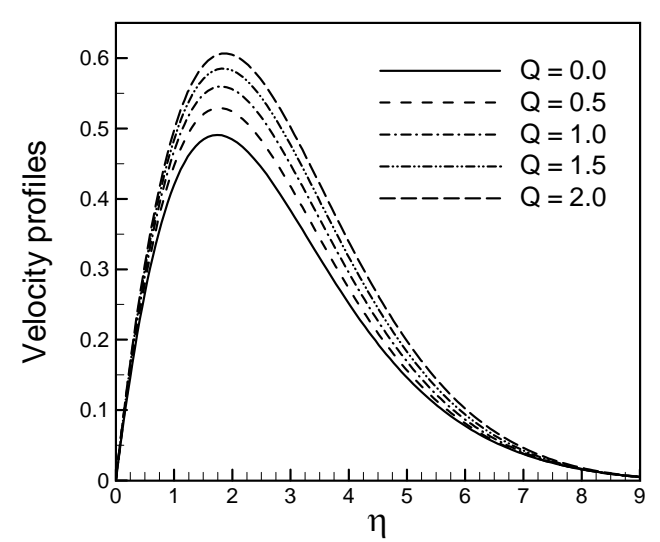

(a)

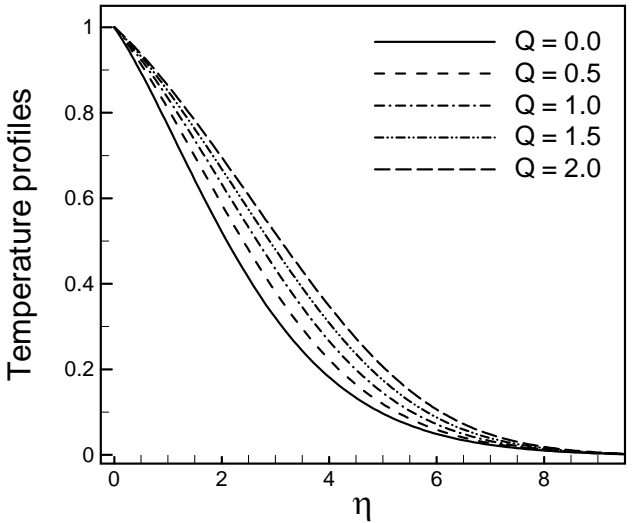

(b)

Figure 4. (a) Velocity and (b) Temperature profiles against $\eta$ for different values of $Q$ with $\operatorname{Pr}=0.72, \alpha=0.2, M=0.1$ and $V d$ $=10.0$.

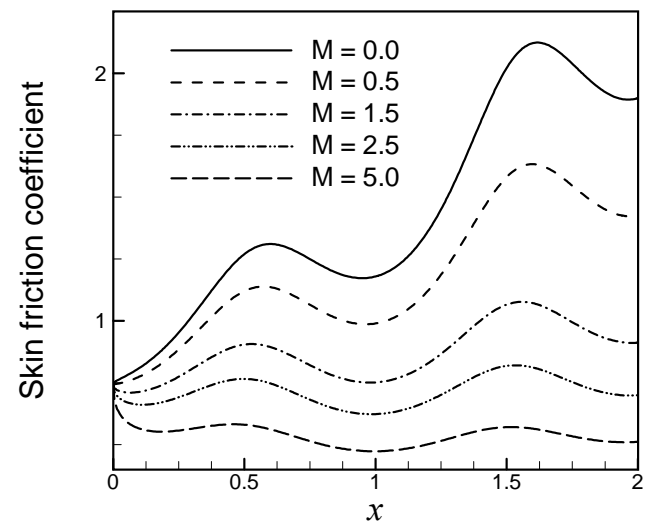

(a)

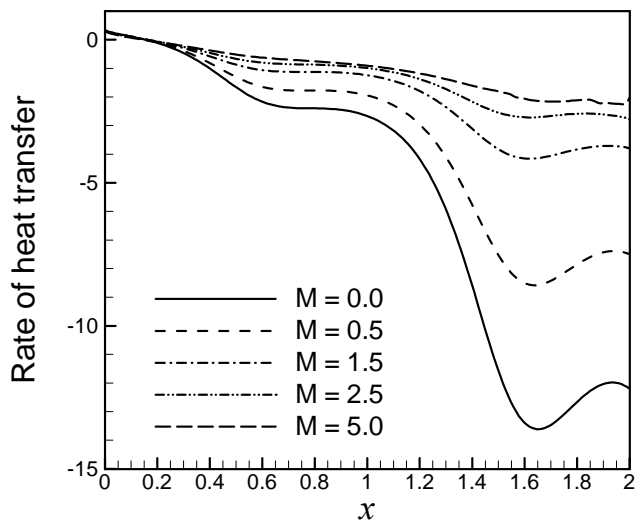

(b)

Figure 5. (a) Skin friction coefficient and (b) Rate of heat transfer against $x$ for different values of $M$ with $\operatorname{Pr}=0.72, \alpha=0.2$, $V d=10.0$ and $Q=0.4$.

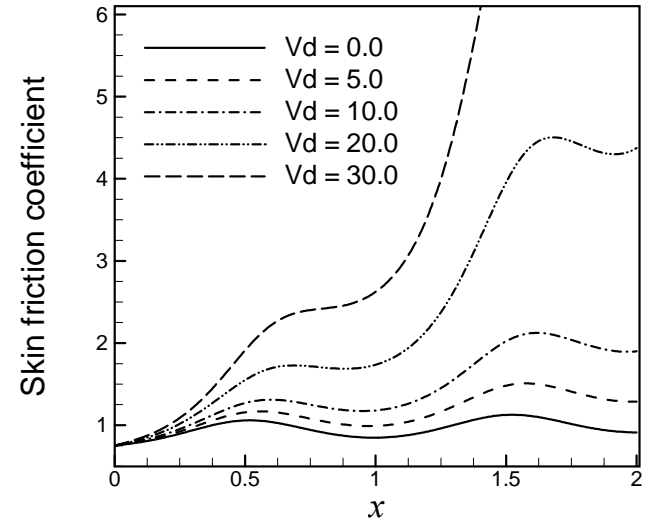

(a)

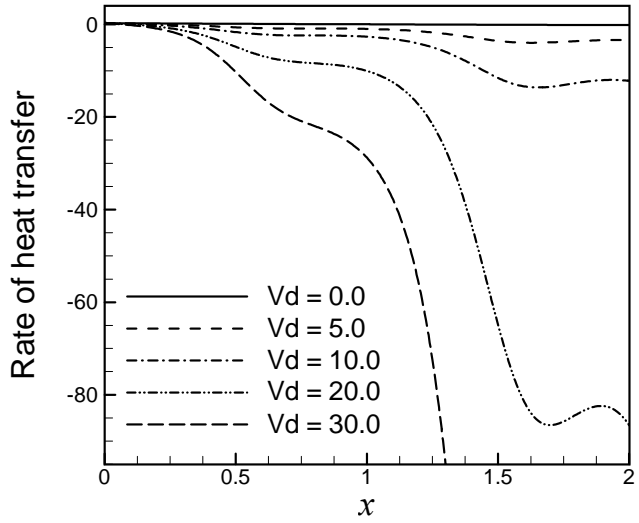

(b)

Figure 6. (a) Skin friction coefficient and (b) Rate of heat transfer against $\mathrm{x}$ for different values of $\mathrm{Vd}$ with $\mathrm{Pr}=0.72, \alpha=0.2$, $M=0.1$ and $Q=0.4$.

therms) distribution shows that temperature decreases significantly as the values of the heat generation parameter $Q$ increases which have been presented in Figure 8(b).
The value of isotherm is 1.0 at the wall and isotherms decreases slowly along the y-direction and finally approach to zero. 


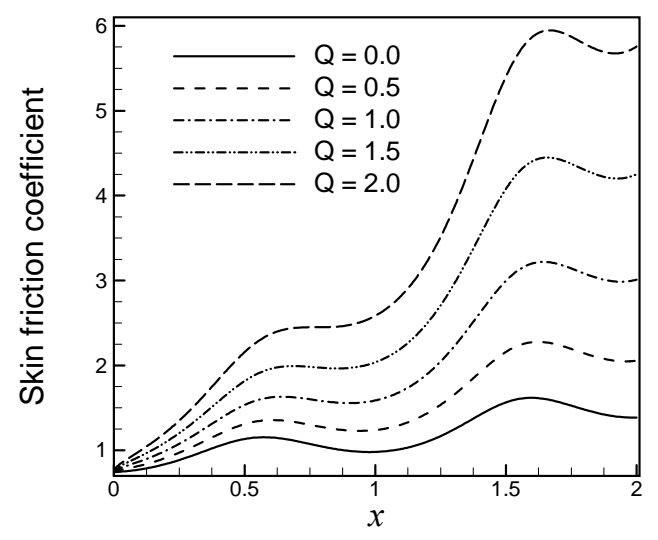

(a)

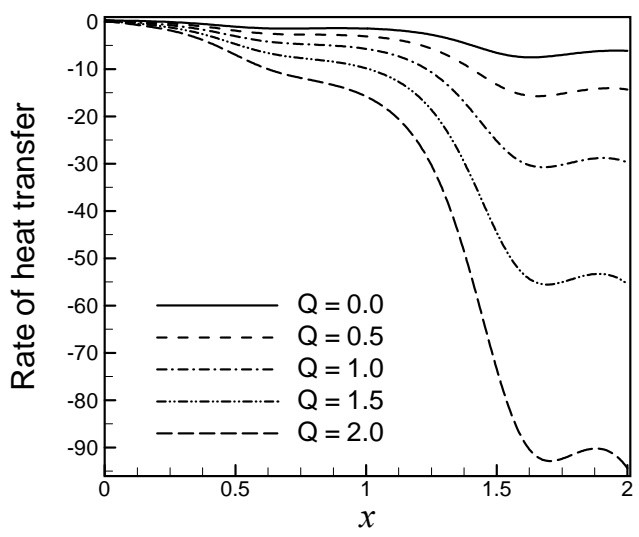

(b)

Figure 7. (a) Skin friction coefficient and (b) Rate of heat transfer against $x$ for different values of $Q$ with $\operatorname{Pr}=0.72, \alpha=0.2$, $M=0.1$ and $V d=10.0$.

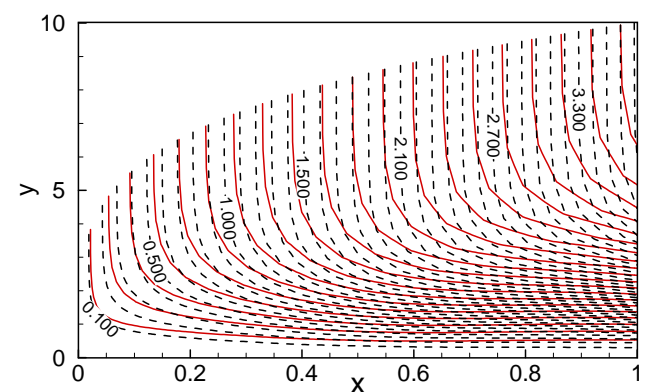

(a)

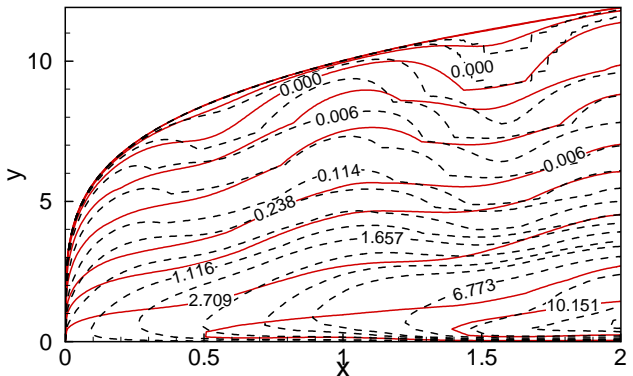

(b)

Figure 8. (a) Streamlines and (b) Isotherms for $Q=0.0$ (Red solid lines), $Q=2.0$ (Black dashed lines), with $\operatorname{Pr}=0.72, \alpha=0.2$, $M=0.1$ and $V d=10.0$.

\section{Conclusions}

The effects of the Prandtl number Pr, the magnetic parameter $M$, the viscous dissipation parameter $V d$, the heat generation parameter $Q$ and the amplitude of wavy surface $\alpha$ on MHD natural convection flow of viscous incompressible fluid along a uniformly heated vertical wavy surface have been studied. From the present investigation the following conclusions may be drawn:

The temperature and the rate of heat transfer coefficient increase for increasing values of magnetic parameter. The velocity decreases and at the position of $\eta=5.5$ becoming constant that is velocity profile meets at the point and then crosses the side and increases with magnetic parameter. The local skin friction coefficient decreases due to the increased value of magnetic parameter.

The velocity and the temperature rise up and the local skin friction coefficient increase due to the higher values of viscous dissipation parameter $V d$ which cause reduction of the rate of heat transfer.

The velocity, temperature and the skin friction coefficient enhance for higher values of internal heat generation parameter $Q$ but for the same reason the rate of heat transfer reduces.

\section{REFERENCES}

[1] L. S. Yao, "Natural Convection along a Vertical Wavy Surface," ASME Journal of Heat Transfer, Vol. 105, No. 3, 1983, pp. 465-468. doi:10.1115/1.3245608

[2] S. G. Moulic and L. S. Yao, "Mixed Convection along Wavy Surface," ASME Journal of Heat Transfer, Vol. 111, No. 4, 1989, pp. 974-979. doi:10.1115/1.3250813

[3] M. A. Alim, S. Alam and M. Miraj, "Effects of Temperature Dependent Thermal Conductivity on Natural Convection Flow along a Vertical Wavy Surface with Heat Generation," International Journal of Engineering \& Technology, Vol. 11, No. 6, 2011, pp. 60-69.

[4] M. A. Hossain and D. A. S. Rees, "Combined Heat and Mass Transfer in Natural Convection Flow from a Vertical Wavy Surface," Acta Mechanica, Vol. 136, No. 3-4, 1999, pp. 133-141. doi:10.1007/BF01179253

[5] C. C. Wang and C. K. Chen, "Transient Force and Free Convection along a Vertical Wavy Surface in Micropolar Fluid," International Journal of Heat and Mass Transfer, Vol. 44, No. 17, 2001, pp. 3241-3251. doi:10.1016/S0017-9310(00)00329-X

[6] K. C. A. Alam, M. A. Hossain and D. A. S. Rees, "Magnetohydrodynamic Free Convection along a Vertical Wavy Surface," Journal of Applied Mechanical Engineering, Vol. 1, 1997, pp. 555-566. 
[7] J. H. Jang, W. M. Yan and H. C. Liu, "Natural Convection Heat and Mass Transfer along a Vertical Wavy Surface," International Journal of Heat Mass Transfer, Vol. 46, No. 6, 2003, pp. 1075-1083. doi:10.1016/S0017-9310(02)00361-7

[8] J. H. Jang and W. M. Yan, "Mixed Convection Heat and Mass Transfer along a Vertical Wavy Surface," International Journal of Heat Mass Transfer, Vol. 47, No. 3, 2004, pp. 419-428. doi:10.1016/j.ijheatmasstransfer.2003.07.020

[9] M. M. Molla, M. A. Hossain and L. S. Yao, "Natural Convection Flow along a Vertical Wavy Surface with Uniform Surface Temperature in Presence of Heat Generation/Absorption," International Journal of Thermal Sciences, Vol. 43, No. 2, 2004, pp. 157-163. doi:10.1016/j.ijthermalsci.2003.04.001

\section{Nomenclature}

$C_{f x} \quad$ Local skin friction coefficient

$C_{p} \quad$ Specific heat at constant pressure $\left[\mathrm{J} \cdot \mathrm{kg}^{-1} \cdot \mathrm{K}^{-1}\right]$

$f \quad$ Dimensionless stream function

$g \quad$ Acceleration due to gravity $\left[\mathrm{ms}^{-2}\right]$

Gr Grashof number

$k \quad$ Thermal conductivity $\left[\mathrm{Wm}^{-1} \cdot \mathrm{K}^{-1}\right.$ ]

$k_{\infty} \quad$ Thermal conductivity of the ambient fluid $\left[\mathrm{Wm}^{-1} \cdot \mathrm{K}^{-1}\right.$ ]

$L \quad$ Characteristic length associated with the wavy surface $[\mathrm{m}]$

$\bar{n}$ Unit normal to the surface

$N u_{x}$ Local Nusselt number

$P \quad$ Pressure of the fluid $\left[\mathrm{Nm}^{-2}\right]$

$\operatorname{Pr}$ Prandtl number

$Q \quad$ Heat generation parameter

$Q_{0} \quad$ Heat generation constant

$q_{w} \quad$ Heat flux at the surface $\left[\mathrm{Wm}^{-2}\right]$

$T$ Temperature of the fluid in the boundary layer [K]

$T_{w} \quad$ Temperature at the surface $[\mathrm{K}]$
[10] B. Tashtoush and M. Al-Odat, "Magnetic Field Effect on Heat and Fluid flow over a Wavy Surface with a Variable Heat Flux," Journal of Magnetism and Magnetic Materials, Vol. 268, No. 3, 2004, pp. 357-363. doi:10.1016/S0304-8853(03)00547-X

[11] M. A. Hossain, S. Kabir and D. S. A. Rees, "Natural Convection of Fluid with Temperature Dependent Viscosity from Heated Vertical Wavy Surface," Zeitschrift für Angewandte Mathematik und Physik, Vol. 53, No. 1, 2002, pp. 48-57. doi:10.1007/s00033-002-8141-Z

[12] N. Parveen and M. A. Alim, "Joule Heating Effect on Magnetohydrodynamic Natural Convection flow along a Vertical Wavy Surface with Viscosity Dependent on Temperature," International Journal of Energy \& Technology, Vol. 3, 2011, pp. 1-10.

$T_{\infty} \quad$ Temperature of the ambient fluid [K]

$u, v$ Dimensionless velocity components along the $(x, y)$ axes $\left[\mathrm{ms}^{-1}\right]$

$x, y$ Axis in the direction along and normal to the tangent of the surface

\section{Greek Symbols}

$\alpha \quad$ Amplitude of the surface waves

$\beta \quad$ Volumetric coefficient of thermal expansion $\left[\mathrm{K}^{-1}\right]$

$\eta \quad$ Dimensionless similarity variable

$\theta$ Dimensionless temperature function

$\psi \quad$ Stream function $\left[\mathrm{m}^{2} \cdot \mathrm{s}^{-1}\right]$

$\mu \quad$ Viscosity of the fluid $\left[\mathrm{kg} \cdot \mathrm{m}^{-1} \cdot \mathrm{s}^{-1}\right]$

$\mu_{\infty} \quad$ Viscosity of the ambient fluid

$v \quad$ Kinematic viscosity $\left[\mathrm{m}^{2} \cdot \mathrm{s}^{-1}\right]$

$\rho \quad$ Density of the fluid $\left[\mathrm{kg} \cdot \mathrm{m}^{-3}\right]$

$\sigma_{0} \quad$ Electrical conductivity

$\tau_{w} \quad$ Shearing stress 